\title{
Comparison between the electrocatalytic properties of different metal ion phthalocyanines and porphyrins towards the oxidation of hydroxide
}

\author{
Karolien De Wael*, Annemie Adriaens \\ Ghent University, Department of Analytical Chemistry, Krijgslaan 281 S12, B-9000 Ghent, Belgium \\ Received 24 July 2007; received in revised form 17 September 2007; accepted 28 September 2007 \\ Available online 2 October 2007
}

\begin{abstract}
This work reports on the electrocatalytic oxidation of hydroxide using different central metal ion phthalocyanines and porphyrins immobilized on gold electrodes. The apparent electrocatalytic activity of cobalt phthalocyanine or porphyrin modified electrodes was found to be the greatest among the present series of metal ion macrocycles investigated. Copper and unmetallated phthalocyanine or porphyrin modified electrodes show no electrocatalytic behaviour towards hydroxide, such as bare gold. A possible mechanism for the enhanced reactivity of cobalt ion macrocycles towards the oxygen evolution is given. It is also stated that the electrocatalytic activity towards an adsorbate involves several aspects, such as the coordination state of the central metal ion, the nature of the ligand, the stability of the complexes, the number of $d$ electrons, the energy of orbitals and the strength of the bonding between the central metal ion and the axial ligand.
\end{abstract}

(c) 2007 Elsevier B.V. All rights reserved.

Keywords: Modified electrode; Electrocatalysis; Phthalocyanines; Porphyrins; Hydroxide; Oxygen evolution

\section{Introduction}

Most studies in electrocatalysis are focused on the electroformation and reduction of oxygen, because of their use in many electrochemical devices such as water electrolysis, fuel cells, ... $[1,2]$. The evolution of oxygen is important in the field of photosynthesis and in the functioning of electrolysers which may by the basis of the production of hydrogen as a clean fuel [3]. The anodic oxygen electrode reaction is known for its high activation overpotential in aqueous solutions resulting in substantial energy losses. Therefore, it is desired to limit the oxygen overvoltages as much as possible.

A series of papers is devoted to the voltammetric determination of hydroxide ions with formation of oxygen in aqueous solutions by using micro-electrodes or rotating disc electrodes [4-6]. In these cases, the concentration of bases can be determined by direct oxidation of hydroxide ions. The potential at which this oxidation occurs varies between 1.1 and $1.4 \mathrm{~V}$ vs.

\footnotetext{
* Corresponding author. Tel.: +32926448 20; fax: +3292644960.

E-mail address: Karolien.DeWael@UGent.be (K. De Wael).
}

SCE. Another approach to reduce the oxygen overvoltages, is the use of electrocatalysts. Some semiconducting oxides, such as $\mathrm{NiCo}_{2} \mathrm{O}_{4}$, give quite good performance towards hydroxide, but the best anodes described in literature seem to be those based on $\mathrm{Ir} / \mathrm{RuO}_{2}$ coatings of $\mathrm{Ti}$ which give current densities at an overpotential of $200 \mathrm{mV}[7,8]$.

As phthalocyanines and porphyrins can act as an electron transfer mediator for several compounds [2], they are potential species for the electroanalytical determination of the $\mathrm{OH}^{-}$ ion concentration. Phthalocyanines and their derivatives have been widely used as dyestuffs and colours. Nowadays they are also commonly used in high-tech applications, such as photovoltaic, electronic and sensing devices [9-12]. Porphyrins are widespread compounds in nature. They are important for several mechanisms in life: they catalyse enzymatic reactions and are, for instance, responsible for the oxygen transport in the human body [13]. These complexes are among the most common and best-known bioinorganic compounds: chlorophylls, cobalamins, hemoglobin, ... [14,15]. The major characteristics of phthalocyanines and porphyrins are their thermal and chemical stability and their extensive redox chemistry. The extensive $\pi$ system of phthalocyanine and porphyrins causes very sta- 
ble complexes with a variety of metal ion. The central metal ion of these macrocycles binds one or two axial ligands and undergoes either metal centered, macrocycle centered and/or axial ligand centered redox reactions. The immobilization of these compounds on an electrode surface can lead to modified electrodes with electrocatalytic properties [16]. Transition metal complexes of macrocyclic ligands, including phthalocyanines and porphyrins, have been widely described as catalysts for oxygen reduction [2]. The use of these compounds for oxygen evolution was already published for carbon electrodes but not for gold [17]. In the former situation, no peak shaped processes was observed thus lacking analytical purposes. The successful use of transition metal ions phthalocyanines and porphyrins as catalysts can be related to the fact that these compounds have unpaired d electrons and unfilled d orbitals which are available to form bonds with the adsorbate in axial position. In the literature, the electrocatalytic activity is most of the time expressed as a function of the number of $d$ electrons of the metal ion in the phthalocyanine or porphyrin ring [2,17-19].

In this paper, we will show that some phthalocyanines and porphyrins immobilized on gold electrodes exhibit enhanced reactivity towards the oxygen evolution. The influence of the nature of the central metal ion on the electrocatalytic behaviour is investigated in detail. It is proven that not only the number of $d$ electrons of the central metal ion is linked to the electrocatalytic activity of the macrocycle. Electrocatalysing the oxidation of hydroxide opens up new perspectives for its analytical application, especially in sensor development to monitor the hydroxide concentration during processing.

\section{Experimental}

A saturated calomel reference electrode (SCE, Radiometer, Copenhagen) with two compartments from Radiometer and a carbon counter electrode were used. The working electrodes were gold electrodes from BASi (West Lafayette, USA) (diameter $1.6 \mathrm{~mm}$ ) and were pretreated by mechanical and electrochemical polishing. Before its first use, the electrode surface was scoured briefly on 1200 grit SiC-emery paper to obtain a fresh surface. To smoothen this relatively rough surface, it was further subjected to sequential polishing on a polishing cloth covered with alumina (Buehler, Illinois, USA) powder of $1,0.3$ and $0.05 \mu \mathrm{m}$ particle size for respectively 5,10 and 20 min. To remove any adherent $\mathrm{Al}_{2} \mathrm{O}_{3}$ particles, the electrode surface was rinsed thoroughly with doubly deionised water and cleaned in an ultrasonic bath (Branson 3210) for $2 \mathrm{~min}$. Finally, the electrode was pretreated electrochemically by scanning it in a $\mathrm{Na}_{2} \mathrm{HPO}_{4} / \mathrm{NaOH}$ buffer solution $(\mathrm{pH} 12$, purchased from Riedel-de Haën) between -1.2 and $0.6 \mathrm{~V}$ vs. SCE until 5 subsequent scans were identical. The scan rate for all cyclic voltammetric experiments was $50 \mathrm{mV} \mathrm{s}^{-1}$.

A PGSTAT20 potentiostat (ECO Chemie, The Netherlands) controlled by GPES 4.9 software package running on a Pentium II computer (Eknadata) was used to obtain the voltammetric data. Measurement of the $\mathrm{pH}$ of the solution was done using a Model 420A Orion Benchtop pH-meter. The phthalocyanine compounds, more specific $\mathrm{Co}(\mathrm{II})$ tetrasulphonated phthalocyanine
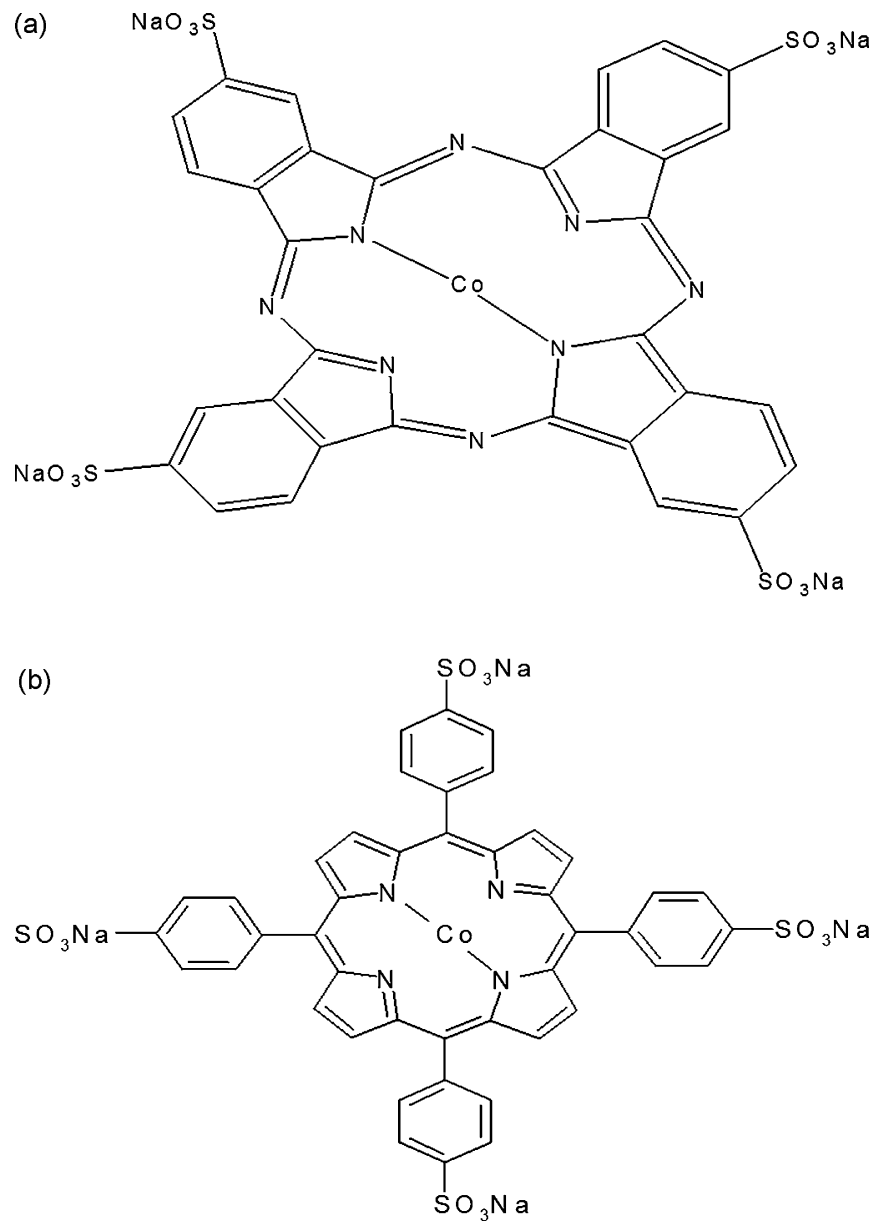

Fig. 1. Molecular structure of $\mathrm{Co}(\mathrm{II})$ tetrasulphonated phthalocyanine sodium salt (CoTSPc) (a) and 5,10,15,20-tetrakis(4-sulphonatophenyl)porphyrin-Co(II) tetrasodium salt (CoTSPor) (b)

sodium salt (CoTSPc, Fig. 1a), 3,4 $4^{\prime}, 4^{\prime \prime}, 4^{\prime \prime \prime} \mathrm{Cu}$ (II) tetrasulphonated phthalocyanine sodium salt (CuTSPc) and Fe(III) tetrasulphonated phthalocyanine sodium salt (FeTSPc) were purchased respectively from Sigma-Aldrich (USA), Rhodes University of Grahamstown, Eastern Cape (South Africa) and Midcentury (Posen, USA). The porphyrin compounds were purchased from Porphyrin Systems (Lübeck, Germany): 5,10,15,20-tetrakis(4-sulphonatophenyl)porphyrin-Co(II) tetrasodium salt (CoTSPor, Fig. 1b), 5, 10,15,20-tetrakis-(4-carboxyphenyl)-porphyrin-Co(II) (CoTCPor), 5,10,15,20-tetrakis(4-carboxyphenyl)-porphyrin-Cu(II) (CuTCPor), 5,10,15,20tetrakis-(4-sulphonatophenyl)-porphyrin-Mn(III) chloride (MnTSPor) and 5,10,15,20-tetrakis-(4-carboxyphenyl)-21,23Hporphyrin $\left(\mathrm{H}_{2} \mathrm{TCP}\right.$ r). Sodium hydroxide $(\mathrm{NaOH})$ and sodium sulphate $\left(\mathrm{Na}_{2} \mathrm{SO}_{4}\right)$ were purchased from Sigma-Aldrich. Before each experiment, pure nitrogen was bubbled through the cell solution for $20 \mathrm{~min}$.

Electrode modification was performed by recording successive cyclic voltammetric scans (60 scans) in a potential window from -1.2 to $0.6 \mathrm{~V}$ vs. SCE in a $\mathrm{Na}_{2} \mathrm{HPO}_{4} / \mathrm{NaOH}$ pH 12 buffer solution containing $1 \times 10^{-3} \mathrm{~mol} \mathrm{~L}^{-1}$ phthalocyanine or porphyrin. After each modification procedure, the electrode was immersed in a $\mathrm{Na}_{2} \mathrm{HPO}_{4} / \mathrm{NaOH}$ pH 12 buffer solution (not con- 
taining phthalocyanine or porphyrin) while voltammetric scans were taken in order to study the electrochemical behaviour of the modified electrode.

\section{Results and discussion}

\subsection{Oxidation of hydroxide at several phthalocyanine or porphyrin modified gold electrodes}

Fig. 2 shows the current-potential behaviour of a bare gold electrode (1) and different phthalocyanine or porphyrin modified gold electrodes (2-9) in a $\mathrm{Na}_{2} \mathrm{HPO}_{4} / \mathrm{NaOH}$ buffer solution containing $0.01 \mathrm{~mol} \mathrm{~L}^{-1}$ hydroxide. A detailed explanation of all electrochemical processes related to the phthalocyanine or porphyrin can be found in literature [20-22]. Only the oxidation process in the potential region of $0.7-1.2 \mathrm{~V}$ vs. SCE and the reduction peak that starts at $-0.1 \mathrm{~V}$ vs. SCE are relevant for this study. The latter is the reduction of dissolved oxygen. As the buffer solution is saturated with nitrogen before and during the electrochemical experiment, the oxygen must be formed during the electrochemical scanning process. It is clear that the oxygen reduction only occurs when there is an oxidation peak in the potential region of $0.7-1.2 \mathrm{~V}$ vs. SCE. This redox reaction can be explained as the electrocatalytic oxidation of hydroxide with formation of oxygen [23]:

$4 \mathrm{OH}^{-} \rightleftarrows \mathrm{O}_{2}+2 \mathrm{H}_{2} \mathrm{O}+4 \mathrm{e}^{-}$

Fig. 2 clearly shows that at a bare gold electrode (1) hydroxide is not electrocatalytically oxidized in the presented potential range. At $1.2 \mathrm{~V}$ vs. SCE, there is an increase in current indicating the beginning of the solvent oxidation and thus representing the limit of the potential range. Analogous cyclic voltammetric scans were recorded for $\mathrm{H}_{2}$ TCPor (2), CuTSPc (3) and CuTCPor (4) modified gold electrodes. None of these curves show redox processes in the potential range from 0.7 to $1.2 \mathrm{~V}$ vs. SCE and thus do not electrocatalyse the oxidation of hydroxide. Curves 5-8 are the first cyclic voltammograms recorded at a CoTSPor (5), CoTCPor (6), CoTSPc (7) and MnTSPor (8) modified gold electrode and clearly exhibit electrocatalytic activity towards hydroxide. The electrocatalytic properties are the best for a CoTCPor modified gold electrode which is shown by the highest current and the largest shift towards less positive potentials. The current-potential behaviour of a FeTSPc

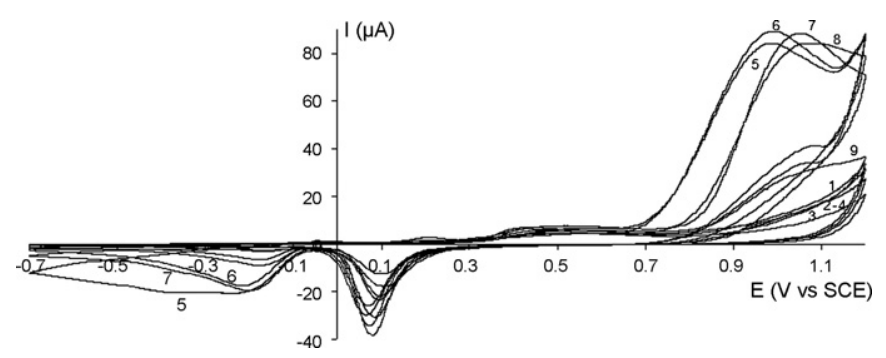

Fig. 2. Cyclic current-potential curves of different modified electrodes in a pH 12 buffer solution: a bare gold electrode (1), $\mathrm{H}_{2}$ TCPor (2), CuTSPc (3), CuTCPor (4), CoTSPor (5), CoTCPor (6), CoTSPc (7), MnTSPor (8) and FeTSPc (9) modified gold electrode. modified electrode is shown as curve 9. Only a poorly defined oxidation peak is observed, indicating a less pronounced electrocatalytic behaviour. The smaller the oxidation peak in the potential region of $0.7-1.2 \mathrm{~V}$ vs. SCE, the less oxygen is formed and thus the lower the reduction current corresponding to the oxygen reduction. The catalytic activity of the modified electrodes towards the oxidation of hydroxide was found to increase as follows:

FeTSPc $<$ MnTSPor $<$ CoTSPc $<$ CoTSPor $<$ CoTCPor (no activity : $\mathrm{Au}, \mathrm{H}_{2}$ TCPor, CuTCPor, CuTSPc)

Based on these observations, it can be concluded that the nature of the central metal ion has a major influence on the electrocatalytic properties of the modified electrode towards hydroxide. A hypothesis for the corresponding mechanism is given further in the text.

Electrocatalysing the oxidation of hydroxide has advantageous consequences for its analytical application, especially in sensor development to monitor the hydroxide concentration during processing. A CoTCPor modified gold electrode is selected to investigate the hydroxide oxidation in detail.

When recording successive cyclic voltammograms at a CoTCPor modified electrode in a $\mathrm{pH} 12$ buffer solution, a decrease in $\mathrm{OH}^{-}$reactivity is observed, as shown in Fig. 3a. Curves 1-6 represent some of the first 20 scans. The decrease of ca. $14 \mu \mathrm{A}$ over 20 scans can be explained by the fact that oxygen formation slowly impairs the immobilized layer, e.g. by the formation of oxo species. The stability was also tested by sweeping the potential from 0 to $0.98 \mathrm{~V}$ vs. SCE (peak maximum) and holding it at $0.98 \mathrm{~V}$ for $760 \mathrm{~s}$ (the time equivalent of measuring 20 successive voltammetric scans). This amperometric experiment is shown in Fig. $3 \mathrm{~b}$ and indicates that the current
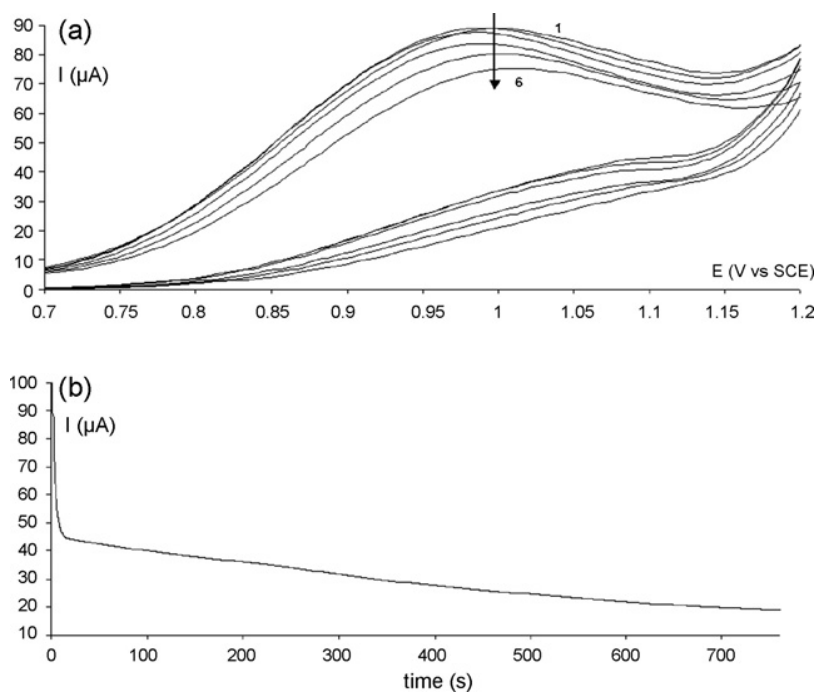

Fig. 3. (a) Successive cyclic voltammograms recorded at a CoTCPor modified electrode in a pH 12 buffer solution: scan 1 (1), 2 (2), 5 (3), 10 (4), 15 (5) and 20 (6); (b) evolution of the current as a function of time for a CoTCPor modified electrode held at a constant potential $(0.98 \mathrm{~V})$ during $760 \mathrm{~s}$ in a $\mathrm{pH} 12$ buffer solution. 

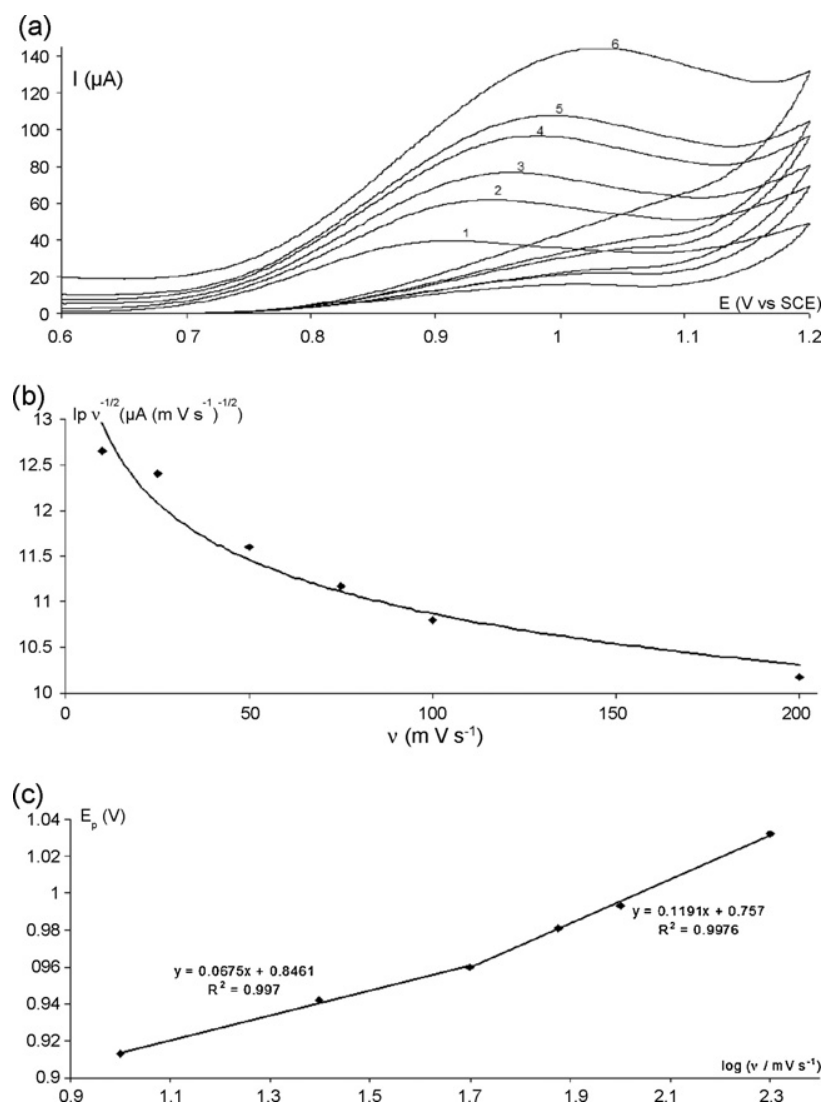

Fig. 4. (a) Oxidation of hydroxide at a CoTCPor modified electrode in a pH 12 buffer solution at different scan rates: $10 \mathrm{mV} \mathrm{s}^{-1}$ (1), $25 \mathrm{mV} \mathrm{s}^{-1}$ (2), $50 \mathrm{mV} \mathrm{s}^{-1}$ (3), $75 \mathrm{mV} \mathrm{s}^{-1}$ (4), $100 \mathrm{mV} \mathrm{s}^{-1}$ (5) and $200 \mathrm{mV} \mathrm{s}^{-1}$ (6); (b) relationship between $I_{\mathrm{p}} v^{-1 / 2}$ and $v$; (c) Relationship between $E_{\mathrm{p}}$ and $\log v$.

declines as a function of time to a much bigger extent compared to the cyclic voltammetric experiment. This decrease is caused by a continuous formation of oxygen during the amperometric experiment. In the limit, the oxygen bubbles will slow down the electron transfer and block the electrode. In contrast, when several successive voltammetric scans are recorded, the formed oxygen is reduced in the cathodic sweep.

The influence of the scan rate on the peak current was investigated to determine the nature of the process. Fig. 4a shows the oxidation of $0.01 \mathrm{~mol} \mathrm{~L}^{-1}$ hydroxide in a $\mathrm{Na}_{2} \mathrm{HPO}_{4} / \mathrm{NaOH}$ buffer solution over a wide range of scan rates. The half-wave potentials shift positively with increasing scan rate, characteristic for an irreversible case. When the slope of the relationship between $\log I_{\mathrm{p}}$ and $\log v$ equals 1 , this corresponds to a reaction of an adsorbed species; while a slope of 0.5 rather indicates a rate determining diffusion of a species towards the electrode surface. For the described redox reaction, a slope of 0.49 is found for the relationship between $\log I_{\mathrm{p}}$ and $\log v$, indicating a reaction of a particle in solution, i.e. hydroxide. In contrast to results obtained at gold micro-electrodes [4], the linearity is valid for all scan rates for modified electrodes. For phthalocyanine and porphyrin modified electrodes, there is no interference between the wave corresponding to the formation of gold oxides with the wave for the hydroxide oxidation. The latter is the case at gold micro-electrodes making the wave due to hydroxide oxidation

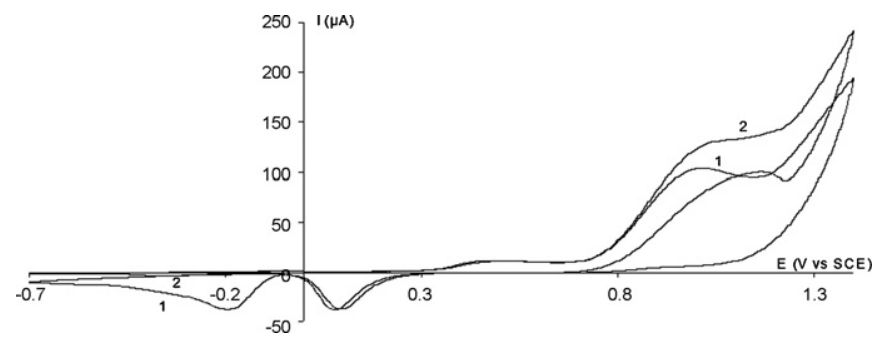

Fig. 5. Cyclic current-potential curves of a stationary (1) and a rotating (2, $76 \mathrm{rpm}$ ) CoTCPor modified electrode in a pH 12 buffer solution.

less defined and no longer useful for analytical purposes at high scan rates [4]. Fig. $4 \mathrm{~b}$ represents the behaviour of $I_{\mathrm{p}} v^{-1 / 2}$ as a function of the scan rate. The $I_{\mathrm{p}} v^{-1 / 2}$ value increases markedly on decreasing the scan rate, characteristic for a catalytic mechanism. The oxidation peak becomes thus less pronounced with decreasing scan rate, indicating an $\mathrm{EC}_{\text {cat }}$ mechanism. This mechanism is usually very readily identified as it is the only one in which this behaviour occurs. Another diagnostic criterion for an $\mathrm{EC}_{\mathrm{cat}}$ mechanism is proposed Fig. 4c: the variation of the peak potential $E_{\mathrm{p}}$ with the scan rate gives two linear regions suggesting that chemical reactions coupled with electrochemical steps are involved [24,25].

The presumed diffusion nature was also tested by varying both the rotation frequency and the concentration of hydroxide ions. For the latter, the reader is referred to the literature [23]. The recorded cyclic voltammograms of a stationary (curve 1) and a rotating (curve 2) CoTCPor modified electrode in a $\mathrm{Na}_{2} \mathrm{HPO}_{4} / \mathrm{NaOH}$ buffer solution, containing $0.01 \mathrm{~mol} \mathrm{~L}^{-1}$ hydroxide, are shown in Fig. 5. In contrast to a stationary electrode, the reduction peak of oxygen at ca. $-0.15 \mathrm{~V}$ vs. SCE disappears when using a rotating electrode as the movement of the electrode moves the oxygen away. It is also observed that the dependence of the limiting current on the rotation speed $\omega$ satisfies the Levich equation [26]. The slope of ca. 0.5 indicates a diffusion controlled process. A value for the diffusion coefficient of $\mathrm{OH}^{-}$ions is calculated: $4.92 \times 10^{-5} \mathrm{~cm}^{2} \mathrm{~s}^{-1}$. Other values reported so far in the literature are: $6.8 \times 10^{-5} \mathrm{~cm}^{2} \mathrm{~s}^{-1}$, determined by Littauer and Tsai [27], in $\mathrm{LiOH}$ solution at infinite dilution by the Pt rde technique, $4.62 \times 10^{-5}$, determined by Daniele et al. [28] and $5.6 \times 10^{-5} \mathrm{~cm}^{2} \mathrm{~s}^{-1}$, determined by Breiter and Hoffmann [29]. In short, the calculated value for the detection limit is in good correlation with the values reported in literature.

\subsection{Interaction between hydroxide and metal ion phthalocyanines or porphyrins}

Only a number of the investigated transition metal ion phthalocyanines or porphyrins immobilized onto a gold electrode exhibit enhanced reactivity towards the oxygen evolution (Fig. 2). The highest activity is observed for 5,10,15,20tetrakis-(4-carboxyphenyl)-porphyrin-Co(II) (CoTCPor). No catalytic activity was observed for unmetallated $\mathrm{H}_{2}$ TCPor: the current-potential behaviour is exactly the same as for a bare, unmodified gold electrode. This shows that ring based redox 
processes are not involved in the electrocatalytic oxidation of hydroxide on phthalocyanine or porphyrin complexes immobilized on gold electrodes. The effect of ring substituents was already investigated in the past [23] and is confirmed in this work. The substituent $\mathrm{COOH}$ yields a slightly better activity compared to $\mathrm{SO}_{3} \mathrm{H}$. The dependence of the kinetics of the reaction on the nature of the central metal ion proofs the idea that the metal ion is the site where the electrocatalytic process occurs. The catalytic activity of phthalocyanine and porphyrin macrocycles is due to their ability to bind ligands axially. Interaction of an adduct, such as hydroxide, is located in an axial position, as a ligand of the metal center of the phthalocyanine or porphyrin ring. In the past, volcano-correlations have been found, when comparing the catalytic activity vs. the redox potential of the metal ion or the number of $d$ electrons of the central metal ion [2,17-19]. It will be shown in this work that more is involved than the redox potential or the number of $d$ electrons.

The cobalt center interacts more favourably with the oxygen atom of the hydroxide ion compared to the other metal ions. According to the perturbation theory, this implies that the orbital energy of cobalt is similar to the one of oxygen [30]. Based on studies concerning the catalytic activity of, on the one side, cobalt macrocycles towards small adducts other than hydroxide and, on the other hand, the electrocatalytic activity of cobalt oxides towards the oxygen evolution [31], the following reaction mechanism is suggested (equatorial ligands are left out) [32]:

$\mathrm{Co}(\mathrm{III})_{\mathrm{ads}}+\mathrm{OH}^{-} \rightleftarrows \mathrm{Co}(\mathrm{III})\left(\mathrm{OH}^{-}\right)_{\mathrm{ads}}$

$\mathrm{Co}(\mathrm{III})\left(\mathrm{OH}^{-}\right)_{\mathrm{ads}} \rightleftarrows\left[\mathrm{Co}(\mathrm{II})(\mathrm{OH})_{\mathrm{ads}}\right]^{-}$

$\left[\mathrm{Co}(\mathrm{II})(\mathrm{OH})_{\mathrm{ads}}\right]^{-} \rightleftarrows \mathrm{Co}(\mathrm{III})(\mathrm{OH})_{\mathrm{ads}}+\mathrm{e}^{-}$

$\mathrm{Co}(\mathrm{III})(\mathrm{OH})_{\mathrm{ads}}+\mathrm{OH}^{-} \rightleftarrows \mathrm{Co}(\mathrm{III})_{\mathrm{s}}+\frac{1}{2} \mathrm{O}_{2}+\mathrm{H}_{2} \mathrm{O}+\mathrm{e}^{-}$

Just before the onset of the oxygen evolution, the oxidation state of the cobalt ion is +III. In the first step, the hydroxide ion occupies the place of the axial ligand of the macrocycle followed by an internal electron shift leading to $\mathrm{Co}(\mathrm{II})(\mathrm{OH})_{\mathrm{ads}}$, which is, because of the applied potential, directly oxidized to $\mathrm{Co}(\mathrm{III})(\mathrm{OH})_{\text {ads }}$ in the third step. This adsorbed species can react with another hydroxide ion resulting in the original $\mathrm{Co}(\mathrm{III})_{\text {ads }}$ state and oxygen evolution. The catalytic oxidation of $\mathrm{OH}^{-}$is due to the stabilization of the intermediate oxidized product through the formation of a covalent bond between the unpaired p electron of hydroxide and an unpaired d electron of the metal ion. This phenomenon is analogous to the one observed in the oxidation of dithiolate-metal complexes via covalent $\mathrm{d}-\mathrm{p}$ bond formation [33]. The extent of this stabilization can be estimated from the negative shift of the redox potential for $\mathrm{OH}^{-}$oxidation in the presence of a transition metal ion phthalocyanine or porphyrin.

Normal $\mathrm{Cu}$ (II) complexes with their $\mathrm{d}^{9}$ configuration have the $d_{x}^{2}-y^{2}$ orbital occupied by the single electron. In $d^{9}$ systems, the octahedral configuration is unstable because of the ambiguity which results from incomplete occupation of the $e_{g}$ orbital. This situation is circumvented through geometrical distortion; a lowering of the symmetry removes the orbital degeneracy (also

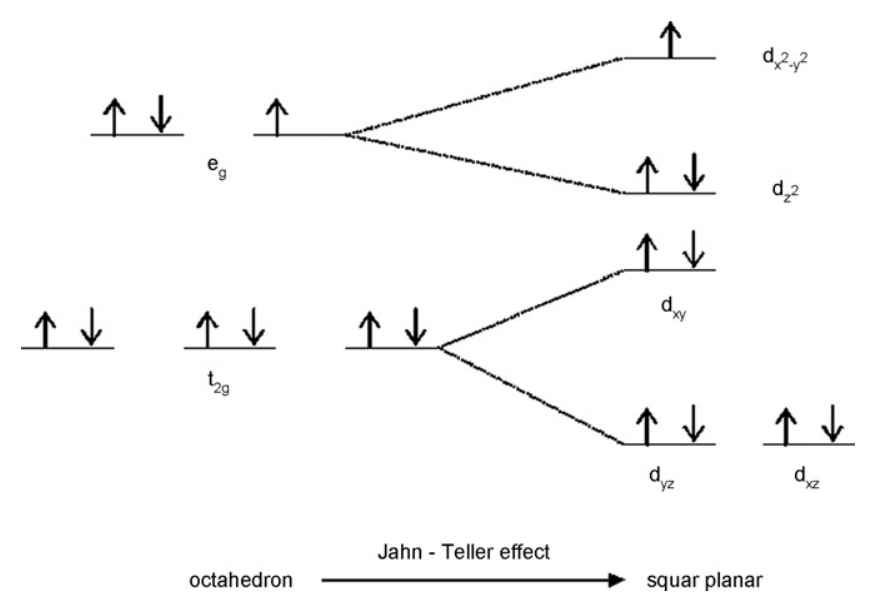

Fig. 6. Jahn-Teller effect of $\mathrm{Cu}(\mathrm{II})$.

called Jahn-Teller effect, Fig. 6). The preference of this metal ion for a square planar geometry and $\mathrm{N}$ ligand coordination, arises from this ligand-field effect [34]. Therefore, the probability for hydroxide to act as an axial ligand is quasi nihil and thus the oxygen evolution will not occur at copper ion phthalocyanines or porphyrins modified electrodes. A second reason for the non-catalytic properties of copper ion phthalocyanines and porphyrins is the fact that the energy of the highest occupied molecular orbital (HOMO) in transition metal ion phthalocyanines and porphyrins decreases almost linearly with the number of d electrons in the metal complex. The energy of the HOMO for copper is probably too low for an interaction between the metal ion and the oxygen. Therefore, a transfer of electron density from an oxygen orbital to d orbitals of the metal is strongly inhibited. A third reason can be derived from the proposed mechanism for hydroxide oxidation. If a hydroxide ion would bind axially towards $\mathrm{Cu}(\mathrm{II})$, in the second step $\mathrm{Cu}(\mathrm{I})$ is formed. This metal ion prefers a tetrahedral geometry [34], a state the rigid phthalocyanine or porphyrin ligand can never adapt. Therefore, further oxidation is excluded. Finally, cobalt, iron and manganese ions are rather hard ions compared to the rather soft nature of a copper ion. Therefore, the former prefer a reaction with a hard ligand, such as hydroxide. In contrast, copper prefers a complexation with a rather soft ligand such as sulfides [34,35].

Based on the hard-soft theory, manganese and iron complexes are expected to show some electrocatalytic behaviour towards hydroxide. Hydroxide is one of the most preferred ligands of $\mathrm{Mn}$ (III) next to phenolate and hydroxamate [34]. This metal ion is known as a powerful oxidizing agent and its relatively fast exchange of hydroxide or oxygen from it. The well-defined catalytic current in Fig. 1 is not a surprise because manganese ions also play an important role in photosynthesis [34]. They show crucial but until now little understood catalytic function in the mechanistically challenging oxidation of water to oxygen. The importance of manganese for the oxygen metabolism is not restricted to photosynthesis; further established examples include a manganese-containing superoxide dismutase, an azide-insensitive catalase and other peroxidases $[36,37]$. 
Iron porphyrin and its analogous derivates are well known because of their stoichiometric oxygen transport in living cells but also in a variety of catalytic biochemical processes [34]. Several small molecules can bind axially towards the iron ion. Fig. 1 shows that Fe(III)TSPc is less active compared to cobalt and manganese complexes, only a small peak can be attributed to the electrocatalytic hydroxide oxidation. This can be explained by the fact that Fe(III)TSPc probably forms a very stable adduct with hydroxide, analogous to Fe(III)EDTA [34]. The release of hydroxide in one of the last steps is slowed down resulting in a lower catalytic activity and thus oxidation peak.

Based on the above results, it is clear that the electrocatalytic activity towards an adsorbate involves lots of aspects, such as the coordination state of the central metal ion, the nature of the ligand, the stability of the complexes, the number of d electrons, the energy of orbitals, the strength of the bonding between the central metal ion and the axial ligand, .... All parameters must be taken into account to describe the catalytic activity.

\section{Conclusions}

This paper describes that a number of metal ion phthalocyanines or porphyrins immobilized on gold electrodes exhibit a catalytic activity towards hydroxide. A detailed electrochemical investigation of the oxidation of hydroxide at these kinds of modified electrodes is performed. It is observed that 5,10,15,20-tetrakis-(4-carboxyphenyl)porphyrin-Co(II) (CoTCPor) appears to be the best electron transfer mediator for this oxidation reaction. A mechanism for the hydroxide oxidation is proposed as well as an explanation for the difference in catalytic activity between different metal ion phthalocyanines and porphyrins. It is clear that the electrocatalytic activity towards an adsorbate, including hydroxide, involves several aspects. All parameters must be taken into account to describe the catalytic activity.

\section{References}

[1] D. Pletcher, Industrial Electrochemistry, Chapman \& Hall, New York, 1982.

[2] J.P. Dodelet, J.H. in, F. Zagal, J.P. Bedioui, Dodelet (Eds.), N4-Macrocyclic Metal Complexes, Springer, New York, 2006 (Chapter 3).

[3] V.P. Shinkarev, C.A. Wraight, Proc. Natl. Acad. Sci. 90 (1993)1834.
[4] M.E. Abdelsalam, G. Denuault, M.A. Baldo, C. Bragato, S. Daniele, Electroanalysis 13 (2001) 289.

[5] O. Ordeig, C.E. Banks, T.J. Davies, F.J. del Campo, F.X. Munõz, R.G. Compton, Anal. Sci. 22 (2006) 679.

[6] A. Abu-Rabi, D. Jasin, S. Mentus, J. Electroanal. Chem. 600 (2007) 364

[7] A.C.C. Tseung, S. Jasem, Electrochim. Acta 22 (1977) 31.

[8] A.J. Scarpellino, G.L. Fisher, J. Electrochem. Soc. 129 (1982) 515, 522.

[9] C.C. Leznoff, A.B.P. Lever, Phthalocyanines Properties and Applications, vol. 3, VCH Publishers, Weinheim, 1993.

[10] A.B.P. Lever, Chemtech 17 (1987) 506-510.

[11] M. Kato, Y. Nishioka, K. Kaifu, K. Kawamura, S. Ohno, Appl. Chem. Lett. 86 (1985) 196

[12] T.A. Temofonte, K.F. Schoch, J. Appl. Chem. 65 (1989) 1350.

[13] K.M. Kadish, K.M. Smith, R. Guilard, The Porphyrin Handbook, Academic Press, New York, 2000.

[14] J.H. Fuhrhop, Angew. Chem. Int. Ed. Engl. 15 (1974) 321.

[15] D. Dolphin, The Porphyrins, vols. I-VII, Academic Press, New York, 1978.

[16] K. De Wael, P. Westbroek, E. Temmerman, Electroanalysis 17 (2005) 263.

[17] N. Chebotareva, T. Nyokong, Electrochim. Acta 42 (1997) 3519.

[18] J.H. Zagal, M. Gulppi, M. Isaacs, G. Cárdenas-Jirón, M.J. Aguirre, Electrochim. Acta 44 (1998) 1349.

[19] J.H. Zagal, S. Lira, S. Ureta-Zañartu, J. Electroanal. Chem. 210 (1986) 95.

[20] K. Peeters, K. De Wael, L. Vincze, A. Adriaens, Anal. Chem. 77 (2005) 5512.

[21] K. De Wael, K. Peeters, D. Bogaert, H. Buschop, L. Vincze, A. Adriaens, J. Electroanal. Chem. 603 (2007) 212

[22] K. De Wael, A. Adriaens, E. Temmerman, Anal. Chim. Acta 554 (2005) 60.

[23] K. De Wael, A. Adriaens, Electrochim. Acta, accepted for publication.

[24] C.A. Caro, F. Bedioui, J.H. Zagal, Electrochim. Acta 47 (2002) 1489.

[25] K.I. Ozoemena, T. Nyokong, J. Electroanal. Chem. 579 (2005) 293.

[26] D. Pletcher, R. Greef, R. Peat, L.M. Peter, J. Robinson, Instrumental Methods in Electrochemistry, Horwood Publishing Limited, England, 2001.

[27] E.L. Littauer, K.C. Tsai, Electrochim. Acta 24 (1979) 351.

[28] S. Daniele, M.A. Baldo, C. Bragato, Anal. Chem. 71 (1999) 811

[29] M. Breiter, K. Hoffmann, Z. Elektrochem. 64 (1960) 462.

[30] R. Hoffmann, Acc. Chem. Res. 4 (1971) 1.

[31] E.B. Castro, C.A. Gervasi, J.R. Vilche, J. Appl. Electrochem. 28 (1998) 835.

[32] C.J. Ballhausen, Introduction to Ligand Field Theory, McGraw-Hill, New York, 1962.

[33] D.T. Sawyer, G.S. Srivatsa, M.E. Bodini, W.P. Schaefer, R.M. Wing, J. Am. Chem. Soc. 108 (1986) 936.

[34] W. Kaim, B. Schwederski, Bioinorganic Chemistry: Inorganic Elements in the Chemistry of Life, second ed., John Wiley \& Sons, New York, 1994.

[35] R.J.P. Williams, Coord. Chem. Rev. 100 (1990) 573.

[36] K. Wieghardt, Angew. Chem. Int. Ed. Engl. 28 (1989) 1153.

[37] G.E.O. Borgstahl, H.E. Parge, M.J. Hickey, W.F. Beyer, R.A. Hallewell, J.A. Tainer, Cell 71 (1992) 107. 